\title{
Citomegalovirus: monitoraggio dell'infezione e della terapia nei pazienti trapiantati di rene
}

\author{
Nicola Liritano', Maria Gabriella Lepore', Rosangela De Grazia', Elio De Fazio', \\ Rosanna Masciari', Gianfranco Merando², Isolina Mantelli², Luigi Lombardi', \\ Paolo Scerbo $^{3}$, Lucio $\mathrm{Cosco}^{3}$, Teresa Ferraro ${ }^{3}$, Caterina Filia ${ }^{4}$, Ignazio Vasapollo ${ }^{4}$
}

\author{
' Virologia e Microbiologia Azienda Ospedaliera Pugliese-Ciaccio-Catanzaro. \\ ${ }^{2}$ Nefrologia e Dialisi Azienda Ospedaliera Pugliese-Ciaccio-Catanzaro. \\ ${ }^{3}$ Malattie Infettive Azienda Ospedaliera Pugliese-Ciaccio-Catanzaro \\ ${ }^{4}$ Virologia e Microbiologia Presidio Ospedaliero Jazzolino-Vibo Valentia
}

Key words: renal transplant, CMV infectious diagnosis, pre-emptive therapy.

\section{SUMMARY}

CMV infection is a major cause of disease following renal transplantation. Clinical diagnosis is difficult because the virus usually produces only few, if any, symptoms.

Consequently rapid and sensitive diagnostic methods are needed, since clinically effective antiviral therapy is available.

Qualitative-quantitative detection of CMV-DNA in leukocytes and in plasma and pp65-antigenaemia are the methods which allow to evidence viral replication activity.

In this study we report the our experience about follow-up of 70 kidney transplant recipients. Results indicate that while active CMV infection occured in 25 patients (37,5\%), only II patients (15,7\%) showed antigenaemia and DNAemia values predictive of CMV disease.

In this study we also evaluated the efficacy of pre-emptive therapy.

\section{INTRODUZIONE E SCOPO}

Il Citomegalovirus (CMV) appartiene alla famiglia Herpetoviridae, sottofamiglia Betaherpesvirinae.

I virus appartenenti a tale famiglia sono caratterizzati dal fatto che l'infezione, una volta esaurito lo stadio acuto, si mantiene nell'organismo allo stadio latente con la possibilità di successive riattivazioni dell'infezione produttiva in condizioni di immunosoppressione.

Nel caso del paziente trapiantato l'infezione da CMV può verificarsi sia come infezione primaria (paziente sieronegativo per IgG anti-CMV in pretrapianto, in cui il virus viene trasmesso tramite l'organo trapiantato o tramite emotrasfusione entrambi provenienti da soggetti positivi per IgG anti-CMV), sia come riattivazione endogena (soggetto sieropositivo per IgG anti-CMV in pretrapianto in cui l'evento è conseguente alla irrinunciabile terapia immunosoppressiva post-trapianto).

Raramente, inoltre, si può verificare una situazione di riattivazione endogena più reinfezione: alla riattivazione del virus nell'organismo del ricevente, a seguito della terapia immunosoppressiva, si associa la trasmissione di un nuovo ceppo virale tramite l'organo trapiantato o emotrasfusioni.

Moltissimi pazienti trapiantati sviluppano un'infezione attiva da CMV e, se trattasi di infezione primaria, la sintomatologia è caratterizzata da una grave infezione disseminata (con febbre, leucopenia, piastrinopenia, aumento degli enzimi epatici, linfoadenopatia) con localizzazione d'organo (retinite, encefalite,epatite, polmonite, enterite, esofagite ecc.).

Il CMV rappresenta dunque una seria minaccia per il buon esito del trapianto (l'infezione virale è certamente implicata nell'attivazione di meccanismi di rigetto) e per la stessa sopravvivenza del soggetto trapiantato. Ne consegue che la precocità della diagnosi di infezione, la terapia antivirale e il monitoraggio della replicazione virale e dell'efficacia della terapia, rappresentano punti nodali nella complessa gestione di tali pazienti. L'obiettivo diagnostico fondamentale, pertanto, non è solo quello di accertare nel decorso del post-trapianto una infezione attiva da CMV (subdola perché spesso asintomatica), bensì quello di ricercare indici di predittività di comparsa della malattia e di progressione della stessa in modo da poter mettere in atto un intervento terapeutico pre-emptive, cioè prima della comparsa della sintomatologia.

Le metodiche usate per il controllo della fase ematica dell'infezione sono rappresentate dalla ricerca in immunofluorescenza (IF) nei nuclei dei leucociti del sangue periferico, della fosfoproteina strutturale pp65 e dalla ricerca quali-quantitativa mediante PCR del DNA nei leucociti e nel plasma. 
Oggi è unanimemente accettato che, per quel che riguarda il dosaggio dell'antigenemia pp65, il dato soglia sopra il quale è opportuno iniziare il trattamento antivirale, anche in assenza di sintomatologia, è compreso tra 50 e 100 cellule positive su 200.000 PMNL osservati. Per quanto riguarda invece la quantificazione del DNA mediante PCR, il numero di copie predittivo di sviluppo di malattia è compreso tra 1.000 e 1.500 / 2x $10^{5}$ leucociti oppure $/ 10 \mu 1$ di sangue.

Nel nostro lavoro riportiamo l'esperienza clinicodiagnostica delle metodiche utilizzate per identificare i pazienti a rischio di malattia valutandone la sensibilità e la specificità e stimiamo la frequenza dell'infezione attiva da CMV e l'efficacia del trattamento terapeutico.

\section{MATERIALI E METODI}

Abbiamo seguito per 4 anni (1999-2002) 70 pazienti trapiantati di rene, $48(68 \%)$ di sesso maschile e $22(32 \%)$ di sesso femminile, di età compresa tra 9 e 64 anni (media 37.4).

14 pazienti $(20 \%)$ hanno ricevuto il rene da vivente e $56(80 \%)$ da cadavere.

Il protocollo diagnostico della fase pre-trapianto si è basato sulla ricerca dei marcatori dei virus epatitici B e C, delle IgG anti CMV, anti-EBV, anti-Toxoplasma ed anti-HHV8 (tabella 1).

Tabella I. Caratteristiche dei 70 pazienti

\begin{tabular}{ll}
\hline ETÃ & 9-64 (MEDIA 37.4) \\
\hline Sesso maschile & $48(68 \%)$ \\
\hline Sesso femminile & $22(32 \%)$ \\
\hline Tx da vivente & $14(20 \%)$ \\
\hline Tx da cadavere & $56(80 \%)$ \\
\hline HbsAg positivi & $2(2.8 \%)$ \\
\hline Anti-HCV positivi & $8(11.4 \%)$ \\
\hline CMV IgG positivi & $70(100 \%)$ \\
\hline EBV IgG positivi & $70(100 \%)$ \\
\hline Toxoplasma IgG positivi & $38(54 \%)$ \\
\hline HHV8 IgG positivi & $68(97 \%)$ \\
\hline
\end{tabular}

Nella fase post-trapianto 58 pazienti su $70(82 \%)$ sono stati trattati con terapia immunosoppressiva triplice (steroidi, inibitori delle calcioneurine e azotioprina o micofenolato o sirolimus; i rimanenti $12(18 \%)$ con terapia immunosoppressiva duplice (steroidi ed inibitori delle calcioneurine). In questa fase il protocollo diagnostico si è basato sulla determinazione semestrale dei marcatori dei virus epatitici $\mathrm{B}$ e $\mathrm{C}$ e della DNAemia di EBV, Toxoplasma ed HHV8.

La ricerca dell'antigenemia pp65 e del CMVDNA qualitativo e quantitativo sono invece state effettuate settimanalmente nel corso dei primi 3 mesi, in seguito mensilmente per altri sei mesi ed infine a regolare scadenza semestrale (eccetto ovviamente in quei pazienti riscontrati a rischio di malattia da CMV).
La ricerca dell'antigenemia è stata effettuata con il kit della ditta Argene commercializzato in Italia dall'Amplimedical.

I passaggi principali previsti dalla metodica sono quattro:

1. separazione dei polimorfonucleati (PMNL) dal sangue periferico con destrano e preparazione dei vetrini;

2. fissazione e permeabilizzazione dei PMNL;

3. rivelazione mediante immunofluorescenza;

4. lettura e quantizzazione del risultato

La ricerca qualitativa- mediante PCR- del DNA di CMV nei leucociti e nel plasma è stata effettuata con il kit della ditta Amplimedical la cui sensibilità è rispettivamente di 100 genomi su 100.000 cellule e di 100 copie $/ \mathrm{ml}$. Il prodotto finale di amplificazione, evidenziabile mediante separazione elettroforetica su gel d'agarosio al 2-4\%, ha le dimensioni di $110 \mathrm{bp}$.

La ricerca quantitativa- mediante PCR - del DNA di CMV nei leucociti e nel plasma è stata effettuata con il kit della ditta Amplimedical; gli intervalli di linearità sono rispettivamente da $100 \mathrm{a}$ 200.000 genomi per 100.000 cellule e da 500 a 1.000 .000 di genomi per $\mathrm{ml}$.

La metodica prevede tre fasi principali:

1. estrazione del DNA da campioni biologici (per esempio leucociti o plasma);

2. amplificazione del DNA estratto e dello Standard interno, sottoposti ad una reazione ciclica di sintesi;

3. rivelazione del DNA amplificato con sonde specifiche per il virus e per lo standard interno: mediante un saggio colorimetrico si misurano i livelli di amplificazione del DNA virale e dello Standard interno ed il rapporto tra le loro densità ottiche permette di risalire alla quantità di genomi virali presenti in origine nel campione.

\section{RISULTATI}

Nella fase pre-trapianto 2 pazienti su $70(2.8 \%)$ sono risultati HBsAg positivi modicamente viremici (in media 34.000 copie $/ \mathrm{ml}$ ); 8 pazienti (11.4\%) positivi per HCV Ab, viremici (in media 582.000 U.I./ml). Tutti i 70 pazienti avevano avuto il contatto primario con il CMV e l'EBV (sieropositività per le relative $\mathrm{IgG}$ ).

Nella fase di studio post-trapianto non sono stati riscontrati nuovi casi di infezione da $\mathrm{HBV}$ ed $\mathrm{HCV}$.

L'infezione attiva da CMV è stata riscontrata in 25 pazienti $(35.7 \%)$ risultati positivi al CMVDNA qualitativo su leucociti, ma alla ricerca del CMV-DNA quantitativo solo 11 pazienti (44\%) su 25 hanno evidenziato una carica virale predittiva di malattia (tabella 2) con diffusione ematica rile- 
vata dalla presenza nel plasma di una media di 4.000 copie $/ \mathrm{ml}$.

$\mathrm{Su}$ questi 25 campioni veniva effettuata anche la ricerca dell'antigenemia pp65 e i dati ottenuti sono riportati nella tabella 3 .

Gli 11 pazienti, 6 di sesso maschile e 5 di sesso femminile, risultati a rischio di malattia avevano un'età media di 31,4 anni (21-64); 10 di loro avevano ricevuto il rene da cadavere, 1 solo da vivente. Nella fase post-trapianto 10 pazienti sono stati sottoposti a triplice terapia immunosoppressiva, 1 a duplice. Nessuno di essi aveva manifestato episodi di rigetto acuto nei dodici mesi precedenti il riscontro di positività al CMV-DNA.

Gli 11 pazienti sono stati tempestivamente trattati con farmaci antivirali e precisamente:

- 8 pazienti con Citovirax alla dose di $3 \mathrm{gr} / \mathrm{die}$ per os per 28 giorni;

- 3 pazienti con Citovirax $5 \mathrm{mg} / \mathrm{Kg}$ e.v. per 21 giorni.

Nei 14 pazienti senza carica virale predittiva di malattia è stata effettuata, per 8 settimane consecutive, la ricerca della carica virale per escluderne significativi incrementi.

Tutti 11 i pazienti in trattamento terapeutico hanno evidenziato, al monitoraggio, la precoce negativizzazione dell'antigenemia pp65, mentre la ricerca del DNA su leucociti si manteneva significativamente positiva per un tempo maggiore.

Gli 8 pazienti trattati per os risultavano, una volta finito il ciclo terapeutico, tutti negativi alla ricerca del CMV-DNA e tali si mantenevano nel follow-up. Dei 3 pazienti trattati per e.v., in 1 si evidenziava negatività del CMV-DNA alla fine del ciclo terapeutico ed al follow-up; in 1 negatività alla fine del ciclo terapeutico ma con recidiva nel corso del follow-up con conseguente perdita della funzionalità dell'organo trapiantato e rientro in emodialisi; ed infine in 1 il CMV-DNA, positivo alla fine del ciclo con Citovirax, si è negativizzato dopo un secondo ciclo, con dose raddoppiata, e tale si è mantenuto nel corso del follow-up.

Il paziente, rientrato in emodialisi dopo appena quattro anni di trapianto, aveva ricevuto il rene dalla madre. Nella fase di post-trapianto era stato necessario sottoporlo ad alcune emotrasfusioni. Nel corso del primo anno dal trapianto le condizioni cliniche del paziente, così come del resto la funzionalità dell'organo trapiantato, erano state sempre soddisfacenti. Successivamente veniva riscontrata un'infezione attiva da CMV con carica virale predittiva di malattia, abbinata ad una forte positività delle IgM anti-CMV. Il paziente veniva quindi sottoposto a terapia con due cicli di Citovirax e.v. (al dosaggio di $5 \mathrm{mg} / \mathrm{Kg}$ per 21 giorni).

Dopo sei mesi dall'inizio del trattamento antivira- le è stato rilevato un incremento progressivo della creatininemia. Ulteriori controlli ematochimici e strumentali non mostravano segni di nefropatia in atto e il dosaggio ematico della CsA era come sempre nella norma (AUC tra 6.000 e 8.000 $\mathrm{ng} . \mathrm{h} / \mathrm{ml}$ ). Pertanto veniva eseguita una biopsia sul rene trapiantato ed il quadro istologico riscontrato era quello caratteristico di una infiammazione interstiziale con tubulite e presenza di inclusioni virali nell'endotelio dei capillari glomerulari testimoniando, tale dato, che il trattamento farmacologico non aveva conseguito alcun risultato. Inoltre l'infezione da CMV era sempre attiva e l'incremento della creatinina si manteneva costante.

\begin{tabular}{ll}
$\begin{array}{l}\text { Tabella } 2 \\
\text { PAZIENTI }\end{array}$ & $\begin{array}{c}\text { CMV DNA QUANTITATIVO SU } \\
\text { LEUCOCITI }\end{array}$ \\
\hline $5(20 \%)$ & Borderline: $I 20$ genomi $/ I 0^{5}$ cellule \\
\hline $4(16 \%)$ & 260 genomi $/ I 0^{5}$ cellule $($ media $)$ \\
\hline $5(20 \%)$ & 450 genomi $/ I 0^{5}$ cellule $($ media $)$ \\
\hline $1 I(44 \%)$ & 2250 genomi $/ I 0^{5}$ cellule $($ media $)$ \\
\hline
\end{tabular}

Tabella 3

\begin{tabular}{|c|c|}
\hline $\begin{array}{l}\text { PAZIENTI POSITIVI } \\
\text { CMV DNA }\end{array}$ & $\begin{array}{l}\text { PAZIENTI POSITIVI } \\
\text { ANTIGENEMIA PP65 }\end{array}$ \\
\hline 5 Borderline: 120 genomi $/ / 0^{5}$ cellule & 0 \\
\hline 4260 genomi/ $/ 0^{5}$ cellule (media) & 0 \\
\hline 5450 genomi/ $10^{5}$ cellule (media) & $\begin{array}{c}3: 35 \text { cellule pos } / 2 \times 10^{5} \\
\text { (media) }\end{array}$ \\
\hline II 2250 genomi//05 cellule (media) & $\begin{array}{l}\text { II:105 cellule pos } / 2 \times 10^{5} \\
\text { (media) }\end{array}$ \\
\hline
\end{tabular}

Tabella 4.

Caratteristiche degli II pazienti a rischio di malattia

\begin{tabular}{ll}
\hline ETA & 2I-64 (MEDIA 3 I.4) \\
\hline Sesso maschile & $6(54 \%)$ \\
\hline Sesso femminile & $5(46 \%)$ \\
\hline Tx da vivente & $\mathrm{I}(9 \%)$ \\
\hline Tx da cadavere & $10(91 \%)$ \\
\hline Anti-HCV positivi & $\mathrm{I}(9 \%)$ \\
\hline Triplice terapia post-trapianto & $10(91 \%)$ \\
\hline Duplice terapia post-trapianto & $\mathrm{I}(9 \%)$ \\
\hline Creatininemia mg\% & $\mathrm{I} .0-1.8$ (medial.27) \\
\hline Episodi di rigetto acuto nell'ultimo anno & 0
\end{tabular}

\section{DISCUSSIONE E CONCLUSIONI}

L'esperienza di questo nostro lavoro e i dati in esso riportati ci consentono di fare una serie di considerazioni sia laboratoristiche che clinicoterapeutiche.

L'amplificazione del DNA di CMV mediante PCR è ormai un'indagine diffusamente utilizzata per il monitoraggio del paziente trapiantato. La PCR offre molti vantaggi:

- elevato valore predittivo;

- capacità di riconoscere l'infezione sistemica con rapidità di esecuzione;

- possibilità di utilizzare campioni congelati 
(aspetto questo non trascurabile se si considera che un servizio diagnostico specializzato si trova oggi a dover far fronte ad una tipologia analitica sempre più diversificata);

- possibilità d'identificazione precoce dei pazienti a rischio di malattia in modo più sensibile dell'antigenemia pp65 specie nei casi in cui i valori della DNAemia sono medio-bassi. Nel ricercare il DNA di CMV nei leucociti bisogna tenere conto che $\mathrm{i}$ dati positivi possono essere imputati a virus latente. L'utilizzo di questa tecnica deve quindi essere esclusivamente quantitativo: un titolo che supera la 500 copie depone per la probabile riattivazione del virus. Riguardo l'antigenemia pp65 è da puntualizzare che ha discreta sensibilità, buona specificità e costi abbastanza contenuti, ma presenta lo svantaggio della tecnica piuttosto laboriosa, dei tempi ristretti di processazione del campione (massimo 4 ore dal prelievo) per evitare la marcata riduzione del numero di cellule positive, e infine della lettura soggettiva dei vetrini.

Inoltre i vari studi effettuati sull'antigenemia pp65 (che per anni ha rappresentato ed in molti centri rappresenta ancora la metodica di riferimento per il monitoraggio del paziente trapiantato), hanno concordemente dimostrato che essa consente di individuare $\mathrm{i}$ pazienti a rischio di malattia 34 giorni prima, mentre gli studi effettuati sulla ricerca del DNA di CMV in PCR hanno invece concordemente dimostrato che tale tecnica consente di individuare $\mathrm{i}$ pazienti a rischio ben 46 giorni prima.

Dalla nostra esperienza appare evidente che il monitoraggio dell'efficacia della terapia antivirale è bene che venga effettuato con la ricerca in PCR del DNA senza ricorrere al test dell'antigenemia che si negativizza sistematicamente prima fornendo quindi un dato clinico-diagnostico fuorviante. Infatti è oramai prassi consolidata considerare negativo il paziente in cui il CMV-DNA risulta non rilevabile in due campioni prelevati a distanza di sette giorni. I nostri dati hanno evidenziato l'opportunità di abbinare la PCR sul plasma a quella su cellule sia per una corretta gestione che per un corretto controllo della diffusione ematica dell'infezione.

Riguardo l'incidenza dell'infezione, malgrado la letteratura più recente confermi che la quasi totalità dei pazienti trapiantati di organo solido vada incontro ad un'infezione attiva da CMV, la nostra percentuale è stata solo del 37.5\% comprendendo, in tale dato, anche gli 11 pazienti con carica virale predittiva di malattia.

Per quel che riguarda il paziente rientrato in trattamento dialitico è lecito supporre, alla luce della rilevante e costante positività delle IgM anti$\mathrm{CMV}$, che si sia trattato di una riattivazione con reinfezione da altro ceppo virale acquisito proba- bilmente in seguito ad emotrasfusione.

L'infezione da CMV ha innescato una crisi di rigetto acuto ed entrambi i virus sono risultati resistenti alla terapia convenzionale. È anche da sottolineare la difficoltà nel trattare questo paziente: la terapia antirigetto incrementa l'immunosoppressione e quindi riduce l'immunosorveglianza sul virus mentre la terapia antivirale, riducendo l'immunosoppressione, può innescare un rigetto acuto. Nei 2 pazienti trapiantati portatori di HBsAg e negli 8 portatori di HCV tutti viremici non abbiamo osservato, in corso di immunosoppressione, alterazioni della funzionalità epatica e aumenti significativi delle cariche virali.

In ultimo possiamo concludere che nella nostra casistica, relativamente ai pazienti in cui i valori di DNAemia erano predittivi di malattia, non è stata riscontrata un'evidente prevalenza di un sesso rispetto all'altro (sei di sesso maschile e cinque di sesso femminile).

\section{BIBLIOGRAFIA}

1. Aitken C, Barrett-Muir W, Millar C, Templeton K, et al. Use of molecular assays in diagnosis and monitoring of cytomegalovirus disease following renal transplantation. J Clin Microbiol 1999; 37: 2804-7.

2. Caliendo AM, Schuurman R, Yen-Lieberman B, et al. Comparison of quantitative and qualitative PCR assays for cytomegalovirus DNA in plasma. J Clin Microbiol 2001; Apr, 39: 1334-8.

3. Emery WC, Sabin CA, Cope AV, et al. Application of viral load kinetics to identify patients who develop cytomegalovirus disease after transplantation. Lancet 2000; 355: 2032-6.

4. Gerna G, Baldanti F, Grossi P, et al. Diagnosis and monitoring of human cytomegalovirus infection in transplant recipients. Reviews in Medical Microbiology 2001; 12: 155-75.

5. Preiser W, Rabenau HF, Vogel JU, Brixner V, Doerr HW. Performance characteristics of an automated PCR assay for the quantification of cytomegalovirus DNA in plasma. J Virol Methods 2002; Mar, 101: 149-57.

6. Rubin RH. Importance of CMV in the transplant population. Transplant Infectious Disease 1999; 1: 3-7.

7. Tong CJ, Cuevas LE, Williams H, Bakran A. Prediction and diagnosis of cytomegalovirus disease in renal transplant recipients using qualitative and quantitative polymerase chain reaction. Transplantation 2000; 69: 985-91.

8. Yen-Lieberman B. Diagnosis of human cytomegalovirus disease. Clinical Microbiology 2000; 22: 105-9.

\section{Rosanna Masciari}

U.O. di Virologia e Microbiologia

Az. Osp. "Pugliese-Ciaccio"

Viale Pio X, 88100 Catanzaro

Tel. e Fax: 0961/883490

E-mail: ros49@libero.it 